
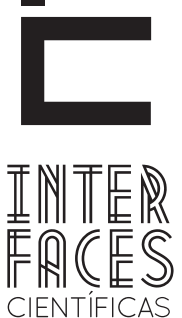

SAÚDE E AMBIENTE

ISSN IMPRESSO 2316-3313

ISSN ELETRÔNICO 2316-3798

DOI - 10.17564/2316-3798.2015v4n1p83-90

\title{
AS CONSEQUÊNCIAS DA DIMINUIÇÃO DE DOPAMINA PRODUZIDA NA SUBSTÂNCIA NIGRA: UMA BREVE REFLEXÃO
}

Madson Alan Maximiano Barreto ${ }^{1}$

Adriana Alves Marinho ${ }^{3}$

Katiuscia Karine Martins da Silva ${ }^{5}$
Andre Fernando de Oliveira Fermoseli ${ }^{2}$

Cícera Lourenço Pontes Fon de Jesus ${ }^{4}$

\section{RESUMO}

Entre os diversos neurotransmissores produzidos no corpo humano temos a dopamina, que é produzida em apenas quatro regiões do cérebro, dentre elas a via nigroestriatal. Esta via é responsável pelo acometimento da Doença de Parkinson, Parkinsonismo, Depressão, entre outras. 0 presente estudo foi feito por meio de uma revisão da literatura sobre a diminuição de dopamina na subtância nigra e as consequências que acometem a terceira idade. Foram pesquisados artigos e livros nos bancos de dados: Scielo, Periódicos CAPES, PUBMED e Biblioteca do Centro Universitário Tiradentes - UNIT. Utilizando os seguintes descritores: Substância nigra, Dopamina, Doença de Parkinson/ Parkinsonismo e Depressão. Dessa forma, este artigo mostra a decorrência da falta de dopamina da via nigroestriatal que tem atingido em maior prevalência indivíduos na terceira idade. A dopamina é um neurotransmissor essencial, assim como qualquer outro, porém, a sua diminuição trará consequências para os indivíduos como o acometimento do Parkinson, Depressão e Parkinsonismo que resultam na diminuição da qualidade de vida do paciente.

\section{PALAVRAS-CHAVES}

Substância nigra, Dopamina, Parkinson/ Parkinsonismo, Depressão. 


\section{ABSTRACT}

Among the many neurotransmitters produced in the human body have dopamine, which is produced in four brain regions, among them the nigrostriatal pathway. This pathway is responsible for onset of Parkinson's Disease, Parkinsonism, depression, among others. This study was done through a literature review on the reduction of dopamine in the nigra substance and consequences that affect the elderly. They were researched articles and books on databases: SciELO, CAPES Journals, PubMed and Library of University Center Tiradentes - UNIT. Using the following keywords: nigra substance, dopamine, Parkinson's Disease / Parkinsonism and depression. Thus, this article shows the re- sult of the lack of dopamine nigrostriatal pathway that has achieved a greater prevalence individuals in old age. Dopamine is an essential neurotransmitter, like any other, but its decline will bring consequences for individuals and the onset of Parkinson's, depression and Parkinsonism that result in the reduction of the patient's quality of life.

\section{KEYWORDS}

Nigra Substance. Dopamine. Parkinson/Parkinsonism. Depression.

\section{RESUMEN}

Entre los muchos neurotransmisores producidos en el cuerpo humano tenemos la dopamina, que se produce en apenas cuatro regiones del cerebro, entre ellas la vía nigroestriatal. Esta vía es responsable de la aparición de la Enfermedad de Parkinson, el parkinsonismo, la depresión, entre otras. Este estudio se realizó a través de una revisión de la literatura sobre la reducción de la dopamina en la sustancia nigra y las consecuencias que afectan a las personas mayores. La investigación fue hecha a través de artículos y libros sobre las bases de datos: Scielo, Revistas CAPES, PUBMED y la Biblioteca del Centro Universitario Tiradentes - UNIT. Utilizando los siguientes descriptores: sustancia nigra, dopamina, enfermedad de Parkinson/parkinsonismo y depresión. Por lo tanto, este ar- tículo muestra el resultado de la falta de dopamina de la vía nigroestriatal que ha tocado con mayor prevalencia a las personas en la tercera edad. La dopamina es un neurotransmisor esencial, como cualquier otro, pero su declive trae consecuencias para los individuos como la aparición de la enfermedad de Parkinson, la depresión y el parkinsonismo que se resultan en la reducción de la calidad de vida del paciente.

\section{PALABRAS CLAVE}

Sustancia Nigra. Dopamina. Parkinson/Parkinsonismo. Depresión. 


\section{INTRODUÇ̄̃̃O}

Este artigo teve como objetivo analisar as consequências causadas pela falta de dopamina produzida na via nigroestriatal, uma vez que algumas das consequências podem ser impedidas se houver conhecimento e compreensão dos profissionais envolvidos nos tratamentos das patologias acometidas nesta região do Sistema Nervoso Central (SNC).

No SNC existem neurônios que liberam neurotransmissores diferentes como a serotonina, GABA, Glutamato, Dopamina e outros. A Sinapse é uma junção entre diversos neurônios agrupados que enviam e recebem estímulos, podendo ser químicos ou elétricos, ocorrendo de um neurônio para outro ou para uma célula. Os neurônios são formados por corpo celular, mitocôndrias, núcleo, dendritos, bainha de mielina, nodo de ranvier, axônio, células de Schwann e terminal do axônio (MACHADO, 2006).

A sinapse ocorre por meio de impulsos que são transmitidos de uma célula nervosa para outra célula, através de sinapses. Normalmente a transmissão é química, e o impulso no axônio pré-sináptico faz com que haja uma liberação de um neurotransmissor no terminal pré-sináptico. Este mediador químico é liberado na fenda sináptica e se liga a receptores específicos na célula pós-sináptica. Em algumas sinapses, a transmissão é puramente elétrica ou puramente química, mas normalmente ela é mista elétrico-química (Figura 1).

Dentre os neurotransmissores, a dopamina (DA) é um neurotransmissor químico produzido em quatro regiões do cérebro humano: nigroestriatal, mesolimbica, mesocortical e túbero-infundibular. Cada uma dessas regiões produtoras de dopamina tem sua função específica, como é o caso da via nigroestriatal, localizada no SNC, mais precisamente no hipotálamo (STANDAERT, 2009).

Uma característica importante da maioria dos neurônios da substância nigra é que eles utilizam como neurotransmissor a dopamina, ou seja, são neurônios dopaminérgicos. As conexões da substância nigra são muito complexas. Entretanto, do ponto de vista funcional, são mais importantes às conexões com o corpo estriado. (MACHADO, 2006, p. 180).

Figura 1 - Neurotransmissão dopaminérgica

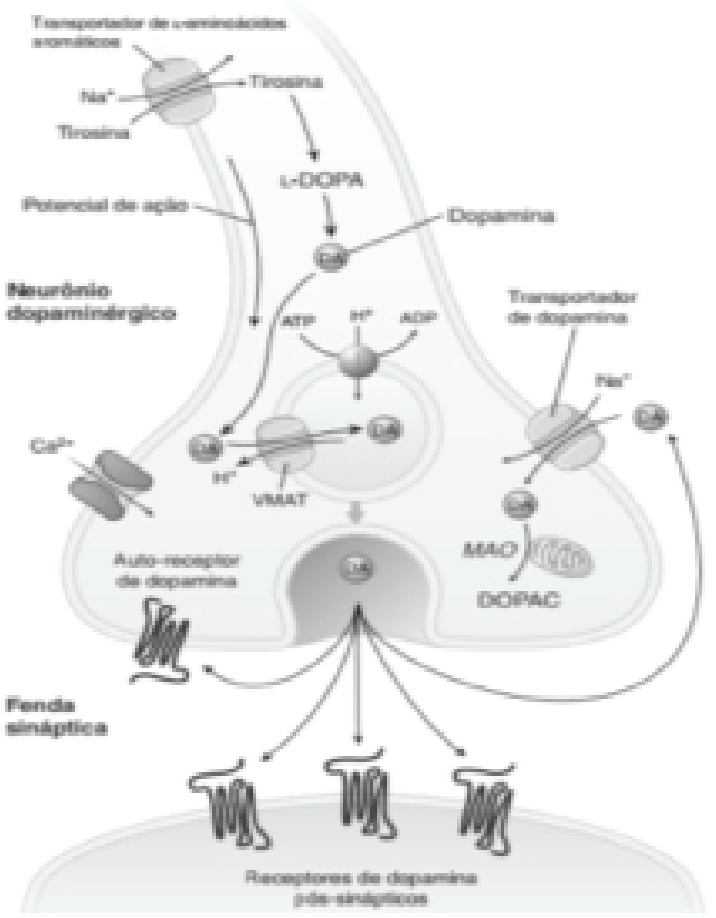

Fonte: STANDAERT e GALANTER (2009, p. 166-185).

Tendo em vista que a DA é produzida em diversas áreas no cérebro, segundo Standaert (2009, p. 169) "[...] o sistema nigroestriatal contém cerca de $80 \%$ [...]", e sua produção em grande quantidade é feita por meio da substância nigra e encaminhada para o estriado, que é formado pelo nucleus acumbens, junto com o caudado e o putamen (CALLEGARO, 2000).

Dentre as funções da dopamina produzida na via nigra estão: controle do movimento, regulação de humor 
e estresse, esquizofrenia e ansiedade. Muitas patologias são ocasionadas com a diminuição da DA nesta via, dificultando o dia a dia dos seres humanos. Como exemplo de doenças apresentadas pela diminuição de DA estão: Doença de Parkinson (DP), Parkinsonismo, Depressão dentre outras (DELUCIA, 2006).

Figura 2 - Localização da via nigriestriatal. Em ampliação uma sinapse normal (acima) e com deficiência de neurotransmissor, P.EX. Dopamina (abaixo)

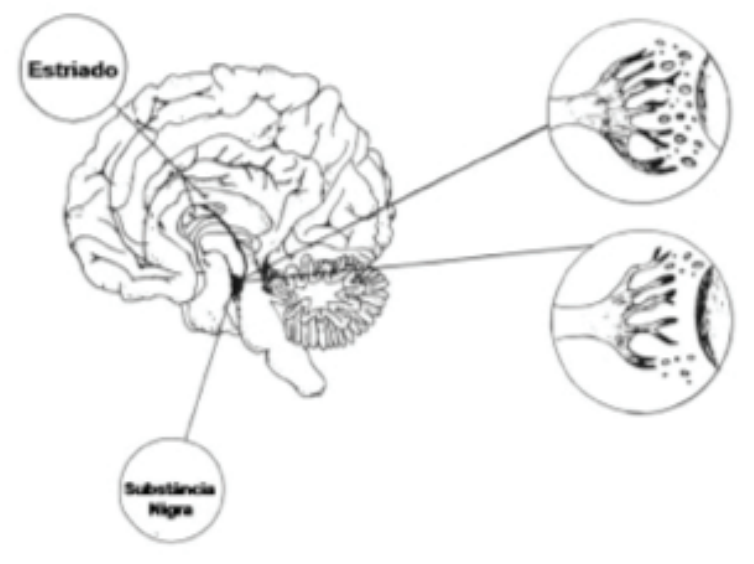

Fonte: Madson Alan Maximiano Barreto.

Portanto, o objetivo deste trabalho é discutir as consequências ocorridas pela diminuição ocasionada pela via nigroestrital, com intuito de mostrar principais patologias apresentada por essa degeneração dada em indivíduos idosos.

\section{METODOLOGIA}

0 presente estudo foi realizado por meio de uma revisão da literatura sobre a diminuição de dopamina na subtância nigra e as consequências para os indivíduos. Foram pesquisados artigos e livros nos bancos de dados: Scielo, Periódicos CAPES, PUBMED e Biblioteca do Centro Universitário Tiradentes - UNIT. Foram utilizados os seguintes descritores: Substância nigra, Dopamina, Parkinson/ Parkinsonismo, Depres- são no período de 2000 a 2014. A seleção inicial foi feita por meio dos títulos e, posteriormente, por meio da leitura de resumos relacionados.

Diante de todos os artigos e livros pesquisados apenas 14 foram utilizados neste artigo. Utilizamos apenas referências que tratavam especificamente das consequências da degeneração da via nigrostriatal relacionados à saúde do paciente.

\section{DECORRÊNCIA DA FALTA DE DOPAMINA NA VIA NIGROESTRIATAL}

A Organização das Nações Unidas (1985) e a Organização Mundial de Saúde (2005) consideram como o início da velhice o momento em que as pessoas atingem 65 anos de idade nos países desenvolvidos e 60 anos de idade para os países em desenvolvimento, como é o caso do Brasil.

Com o passar dos anos ocorre à diminuição de neurônios, contudo, para que todos os indivíduos viessem a ter DP (por exemplo), por uma causa de degeneração natural, isto só ocorreria a partir dos seus 70 anos, quando haveria uma perda significativa destas células, fazendo com que ocorresse o aparecimento não só da DP, mas de tantas outras doenças causadas pela degeneração (FERRAZ, 2012).

A Doença de Parkinson (DP) foi descrita, em 1817, pelo médico James Parkinson, com os seguintes sintomas: presença de tremor, rigidez, bradicinesia e instabilidade postural (ANDRADE, 2004, p. 349). Ao falar destas doenças, muitos desconhecem quais são os seus sintomas, tendo em vista que eles "são comuns a outras doenças degenerativas da idade" (LENT, 2010, p. 69).

A DP, também, pode ocorrer em crianças ou jovens adultos, porém passa a ser considerada não degenerativa da substância nigra no tempo comum, mas sim hereditária (PINEL, 2005). 
O Parkinsonismo, também chamado de Parkinson secundário, tem os mesmos sintomas que a DP, porém essa patologia surge como uma comorbidade causada por alguma doença primária, como por exemplo, o Acidente Vascular Cerebral (AVC). O Parkinsonismo pode ocorrer em qualquer idade, com maior frequência em jovens adultos e, diferente da DP, apresenta etiologia, sendo um dos seus fatores de risco a exposição a drogas bloqueadoras (como dopaminérgicas, antipsicóticos, metoclopramida), exposição a toxinas, traumatismo craniano, entre outros, fazendo com que acarrete esses efeitos degenerativos. Para diagnosticar um indivíduo com Parkinsonismo, é necessário fazer uma ressonância magnética (PEREIRA ET AL., APUD STEIDL, 2007).

Muitos confundem a DP com Parkinsonismo, já que ambas possuem os mesmo sintomas, porém a diferenciação é feita por meio da ressonância magnética (RM) no indivíduo: pacientes portadores de DP não apresentam nenhuma alteração no exame de RM; já o Parkinsonismo mostrará todas as alterações sobre as informações dadas na RM. O exame mostra algumas alterações como acidente vascular encefálico nos núcleos da base ou calcificações, tumores e outros (FERRAZ, 2012).

O quadro da Doença de Parkinson (DP) e Parkinsonismo normalmente acarreta o aparecimento de depressão, ocasionada provavelmente pela falta de dopamina no organismo. Entre as quatro regiões produtoras de dopamina no cérebro, a que está ligada a depressão é a via da substância nigra, localizada no hipotálamo (NAVARRO-PETERNELLA, 2012).

Muitos dos pacientes que apresentam depressão podem desencadear o Transtorno de Depressão Maior que vem descrito no DSM-5 (FUENTES, 2014).

Depressão é constituída por uma desordem mental que ocorre com frequência em idosos e tem causado impactos na qualidade de vida. Desta forma, a depressão vem sendo considerada um fator de risco desencadeador dos processos demenciais (STELLA, 2002).

O diagnóstico da depressão passa por várias etapas: anamnese detalhada, com o paciente e com familiares ou cuidadores, exame psiquiátrico minucioso, exame clínico geral, avaliação neurológica, identificação de efeitos adversos de medicamentos, exames laboratoriais e de neuroimagem. Estes são procedimentos preciosos para o diagnóstico da depressão, intervenção psicofarmacológica e prognóstico, especialmente em função da maior prevalência de comorbidades e do maior risco de morte. (STELLA, 2002, p. 92).

Este transtorno pode ser um dos primeiros fatores já existentes nos pacientes, mesmo antes do diagnóstico de DP ter sido estabelecido. Assim sendo, a suspeita de que estes pacientes já nasçam com déficits dopaminérgicos é algo a ser analisado (FUENTES, 2014).

Muitos dos Pacientes que apresentam DP, Parkinsonismo ou Depressão são indivíduos com idade avançada. Com o acometimento dessas patologias, os pacientes passam a depender de parentes, fazendo com que haja uma desestruturação no funcionamento dos cuidadores, dado que os pacientes passarão a depender da família e, quando não têm suporte para lidar com as demandas apresentadas pelas consequências das patologias, é necessário um trabalho com diversos profissionais da saúde desde o momento do diagnóstico, destacando-se a importância da atuação do psicólogo nos tratamentos dessas doenças no auxilio aos cuidadores (DE ANDRADE, 2003).

Algumas dessas doenças não apresentam etiologia, uma vez que ocorrem por fatores ambientais e genéticos, porém todas têm semelhanças na ocorrência da diminuição do neurotransmissor DA.

Embora diversos profissionais de saúde trabalhem com pacientes que apresentam essas patologias descritas acima, muitos deles não têm conhecimento quanto às consequências causadas pela diminuição não só da DA, mas também de outros neurotransmissores (RODRIGUES, 2006). 
É necessário que os profissionais envolvidos neste contexto se atentem as novas técnicas de imagem e as novas descobertas de processos neuropsicológicos, afim de se entender a patologia de forma interdisciplinar e adequar um tratamento mais eficiente, visando, principalmente a qualidade de vida do paciente.

\section{CONCLUSÃO}

Por fim, percebemos que a dopamina tem uma importância bastante significativa para o ser humano, pois sua falta ou diminuição pode trazer severas consequências, como o surgimento do Parkinson, Parkinsonismo e Depressão, além de outras patologias como a esquizofrenia que está ligada de forma indireta ao parkinsonismo, comportamento emocional, déficit de memória, desencadeamento de estresse que estão ligadas às outras vias produtora de dopamina.

A partir da degeneração destes neurônios, os pacientes portadores da DP, Parkinsonismo e Depressão enfrentam problemas sociais e tendem a ter uma diminuição na expectativa de vida. Tendo em vista que o número de idosos inseridos na sociedade vem aumentando a cada ano, é necessário se ter um olhar para a manutenção da saúde dessas pessoas, fazendo com que não haja a diminuição na perspectiva de vida desses indivíduos.

Em suma, é importante que todos os profissionais envolvidos no tratamento das patologias ocorridas pela degeneração da via nigroestriatal tenham conhecimento das reais decorrências desta, o que possibilitará a elaboração de estratégias para melhor conduzir o tratamento.

\section{REFERÊNCIAS}

DA SILVA, Regina Cláudia Barbosa. Esquizofrenia: uma revisão. Psicologia USP, v.17, n.4, 2006. p. 263-285.
DE ANDRADE, Rosângela Vieira et al. Atuação dos neurotransmissores na depressão. Sistema Nervoso, v.2, 2003. p.3-9.

DELUCIA, Roberto et al. Capítulo 28 Fármacos Usados no Tratamento de Doenças Neurodegenerativ as. Farmacologia Integrada, 2006. p.279- 286.

\section{FERRAZ, B, H. BORGES, V. Como diagnosticar e}

Tratar doença de Parkinson. Disponível em: <http:// www.moreirajr.com.br/revistas.asp?fase=r003\&id materia=1870 >. Acesso em: 17 maio 2015.

FUENTES, D. NEUROPSICOLOGIA: Teoria e Prática. 2.ed, Porto Alegre: Artmed, 2014. p.53-56.

MACHADO, A. Neuroanatomia Funcional. 2.ed. São

Paulo: Atheneu, 2006. p.180-250.

NAVARRO-PETERNELLA, M, F. MARCON, S, S. Qualidade de vida de indivíduos com Parkinson e sua relação com tempo de evolução e gravidade da doença. Rev. Latino-Am. Enfermagem. 2012. Disponivel em: <http://www.scielo.br/pdf/rlae/ v20n2/pt_23.pdf>. Acesso em: 26 jun. 2015.

OIE SALLES, Jerusa Fumagalli. Avaliação neuropsicológica nas doenças neurológicas. Rotinas em neurologia e neurocirurgia, 2008. p.218.

OGANIZAÇÃO MUNDIAL DA SAÚDE (OMS) Organização Pan-Americana da Saúde (OPAS): Resolução CE122.R9, 1998. Saúde das pessoas idosas, 2005.

PINEL. P, J. Biopsicologia. 5.ed. São Paulo: Artmed, 2005. p. 260-269.

ROCHA, Lorena Priscila Oliveira; DE SOUZA CIOFFI, Andreia Correia. Caracterização da depressão entre idosos. Revista Eletrônica Interdisciplinar, v.2, n.12, 2014. 
RODRIGUES, M.; CAMPOS, C. L. estratégia para o tratamento com levodopa na Doença de Parkinson. Departamento de Farmácia Universidade do Extremo Sul Catarinense. Revista Analytica, n.23, Santa Catarina, junho/julho 2006.

STANDAERT, D.; GALANTER, Joshua M. Farmacologia da Neurotransmissão Dopaminérgica. Princípios de Farmacologia: A Base Fisiopatologia da Farmacoterapia, 2009, p.166-185.
STEIDL, S, M, E. Z, R, J. F, V, F. Doença de Parkinson: Revisão bibliográfica. Ciências da Saúde, Santa Maria, v.8, n.1, 2007. p.115-129.

STELLA, Florindo et al. Depressão no idoso: diagnóstico, tratamento e benefícios da atividade física. Motriz, v.8, n.3, 2002. p.91-98. 
1. Graduando do Curso de Psicologia do Centro Universitário Tiradentes UNIT/AL. E-mail: mmaximiano.mm@gmail.com

2. Professor do Curso de Psicologia do Centro Universitário Tiradentes UNIT/AL. E-mail: afermoseli@hotmail.com

3. Professora do Curso de Psicologia do Centro Universitário Tiradentes UNIT/AL. E-mail: adrianaalvesmarinho@hotmail.com

4. Médica Neurologista, fundadora e presidente da Associação Parkinson Alagoas - AsPAL.

5. Psicóloga especialista em Neuropsicologia e em Psicoterapia cognitivoAceito em: 13 de Agosto de 2015 -comportamental. 